\title{
The Relationship of Anticipated Pain and Fear Avoidance Beliefs to Outcome in Patients With Chronic Low Back Pain Who Are Not Receiving Workers' Compensation
}

\author{
Saud M. Al-Obaidi, PhD, MCSP, PT, ${ }^{*}$ Paul Beattie, PhD, PT, OCS,† Baker Al-Zoabi, MSc, PT, \\ and Sami Al-Wekeel, BSc, PT‡
}

Study Design. A prospective, interventional case series design.

Objectives. To determine the degree to which preintervention measures of anticipated pain and fear avoidance beliefs predict outcome after intervention for patients with delayed recovery from low back pain (LBP) for which they are not receiving workers' compensation.

Summary of Background Data. Anticipated pain and fear avoidance beliefs have been suggested as important factors for the classification and treatment of patients with LBP. However, the degree to which they are associated with outcome after intervention is uncertain.

Methods. There were 42 subjects with activity limiting LBP for more than 2 months enrolled in an exercise based, physical therapy program. A multidimensional test battery was completed before and after a 10-week program of lumbar extensor muscle strengthening. Correlational analyses, independent $t$ tests, and validity indexes were used to determine relationships of preintervention measures of anticipated pain and the fear avoidance beliefs to clinically meaningful improvements in the Roland-Morris score. Intention-to-treat strategies were used to account for study dropouts.

Results. A total of 36 subjects completed the 10 -week intervention. The lack of clinically meaningful outcome, as determined by a failure-to-report a minimum of $16 \%$ decrease in the Roland-Morris score, was associated with high preintervention scores on the physical activity subscale of the Fear Avoidance Beliefs Questionnaire (FABQP). Subjects with a preintervention score $\geq 29$ on the FABQP had a likelihood ratio of 3.78 (95\% confidence interval [CI] 2.4-5.16) for an increased probability of negative outcome after initial testing when compared to those subjects with low $(<20)$ scores. The sensitivity and specificity of low scores to predict clinically meaningful outcomes compared to those with high scores were mod-

From the *Department of Physical Therapy, Kuwait University, Faculty of Allied Health Sciences and Nursing, Sulaibikhat, Kuwait; †Program in Physical Therapy, Department of Exercise Science, School of Public Health, University of South Carolina, Columbia, SC; and $\ddagger$ Department of Physical Therapy, Al-Razie Hospital, Ministry of Health, Kuwait City, Kuwait.

Acknowledgment date: November 14, 2003. First revision date: March 11, 2004. Acceptance date: June 15, 2004.

The manuscript submitted does not contain information about medical device(s)/drug(s).

No funds were received in support of this work. No benefits in any form have been or will be received from a commercial party related directly or indirectly to the subject of this manuscript.

Address correspondence and reprint requests to Paul Beattie, PhD, PT, OCS, Program in Physical Therapy, Department of Exercise Science, School of Public Health, University of South Carolina, Columbia, SC 29206; E-mail: pbeattie@gwm.sc.edu erate (sensitivity $=0.87$ and specificity 0.77 ); however, the likelihood ratio was inconclusive. Anticipated pain was significantly correlated with but was higher than the reported pain during activity both before and after intervention but not predictive of outcome.

Conclusions. In a sample of people from a Middle Eastern culture undergoing exercise intervention for LBP for which they are not receiving workers' compensation, the preintervention physical activity subscale of the FABO is predictive of negative outcome when the observed scores are $\geq 29$. Despite significant improvements in all variables after intervention, anticipated pain remained significantly higher than reported pain during physical performance testing but did not predict outcome.

Key words: back pain, outcome, anticipation of pain, fear avoidance beliefs, pain measurement, rehabilitation, cognitive-behavioral variables. Spine 2005;30:1051-1057

The symptoms and disability associated with low back pain (LBP) are caused by an interplay of numerous pathophysiologic and biobehavioral variables. ${ }^{1-5}$ When compared to individuals without symptoms, patients with chronic LBP have had reductions in the crosssectional area and force generating capacity of lumbar muscles, ${ }^{6-8}$ slower walking speed, ${ }^{2}$ and less lumbar range of motion. ${ }^{9,10}$ However, recent literature has suggested that in many instances, biobehavioral factors explain a larger portion of the variance in a patient's clinical picture than do measures of physical impairment. ${ }^{3-5}$ Therefore, it is important to obtain measures of biobehavioral factors during clinical examination.

During the last decade, conceptualization of pain rather than the actual intensity of perceived pain has been proposed as a primary predictor of outcome in patients with acute and chronic LBP. ${ }^{2-5,11-26}$ Two primary ways in which pain can be conceptualized in a negative way are by adverse fear avoidance beliefs and by high degrees of anticipated pain before activity. ${ }^{1-3,11,12,18,20-27}$ When measured in high levels, these factors are predictive of poor physical performance and/or delayed recovery. 1,2,12,23-27

Fear avoidance beliefs and anticipated pain are typically assessed using standardized self-report measures. The Fear Avoidance Beliefs Questionnaire (FABQ) described by Waddell et $a^{23}$ contains 2 subscales: avoidance beliefs related to physical activity, and avoidance beliefs related to work. This instrument has been widely used and yields measures that have evidence of reliability. Measures from the work subscale of the FABQ have 
predictive validity to classify injured workers at increased risk for delayed recovery from LBP. ${ }^{24,25}$ However, the validity of either subscale of the FABQ is less well established in the population of people who are not injured workers.

Recent work has suggested that anticipated pain before physical activity is also an important variable, and may strongly influence a patient's physical performance as well as beliefs regarding his or her overall status. ${ }^{1,2}$ For example, patients with high degrees of anticipated pain may avoid activity or perform tasks less vigorously, which, over time, may lead to physical deconditioning that further enhances disability. In previous research, we have noted relationships of anticipated pain and fear avoidance beliefs to impaired activity performance, and have postulated that both of these measures are clinically relevant for patient classification. ${ }^{1,2}$

The purpose of the present study was to determine the degree to which preintervention measures from the subscales of the FABQ and numerical rating scale measures of anticipated pain predict meaningful improvement in the Roland-Morris Score after intervention for patients with delayed recovery from acute LBP who are not receiving workers' compensation. It was our belief that in a population of people who were not injured workers, the physical activity subscale of the FABQ would be more predictive of outcome than the work subscale. In addition, we believed that anticipated pain would not be highly correlated with either of the subscales of the FABQ, indicating that they represent a different construct.

\section{- Materials and Methods}

Subjects. To be eligible for participation, subjects had to have been referred to physical therapy for evaluation and treatment of activity limiting LBP that had been present for at least 2 months and was not associated with a workers' compensation claim. The presence of associated lower extremity pain was not an exclusion criterion. Subjects could not have had previous physical therapy treatment for LBP. Before entry, all subjects had undergone an evaluation by an orthopedist, neurologist, or neurosurgeon to exclude surgical indications or nonmusculoskeletal sources of symptoms. Subjects were excluded if there was evidence of acute vertebral fracture, Grade II or higher spondylolisthesis, and/or signs of neurologic conductive impairment (lower extremity motor or sensory loss, abnormal deep tendon reflex). Additional exclusion criteria included: cardiovascular or cardiopulmonary conditions that required exercise restriction; the current use of tobacco; and/or the current use of corticosteroids, opiate based analgesics, or psychotropic medications. All subjects were recruited from 4 physical therapy outpatient clinics in Kuwait City, Kuwait, from 2002 to 2003. Before entry into the study, all subjects signed an institutionally approved consent form.

Measures. In addition to demographic reports, the following measures were obtained before and 1 week after intervention. The first author (S.M.A.) obtained all measurements.
Fear-Avoidance Beliefs. The subject's beliefs regarding adverse consequences associated with physical activities and work were measured by a precise Arabic translation of the 2 subscales of the FABQ ${ }^{23}$ Items of both subscales are rated on a 7 -point scale. The end range anchor terms are "completely disagree" ( 0 points) and "strongly agree" (6 points). The middle anchor term is "unsure." The physical activity subscale (FABQP) contains 5 questions relating to fear-avoidance beliefs about physical activity. The total score from this subscale can range from the lowest of 0 to the highest of 30 . The second subscale, the work subscale of the FABQ(W), contains 16 questions regarding fear-avoidance beliefs about work. Scores on this subscale range from the lowest of 0 to the highest of 96 .

Pain Intensity. Pain measures were obtained for 3 conditions: (1) "overall pain" that measured the subject's typical pain throughout the day, (2) "anticipated pain (AP)" that measured the subject's predicted pain during a future activity, and (3) “actual reported pain" that measured a subject's current pain while performing a specific activity. A $100-\mathrm{mm}$ visual analogue scale (VAS) was used to obtain each of these measures. The anchor terms on the VAS for overall pain and actual reported pain were: 0 for "no pain" and 100 for "maximum pain imaginable"; and for anticipated pain were: 0 for "no pain anticipated" and 100 for "maximum anticipated pain." $1,2,28,29$

Physical Performance Tests. Three timed tests were performed: sit to stand, lumbar forward bending, and fast walking (Table 1). The subjects were instructed to complete these performance tasks as fast as possible within pain tolerance. These tests have yielded reliable data and have been advocated as useful clinical measures of various commonly performed tasks. ${ }^{30}$

Roland-Morris Back Pain Disability Questionnaire. Perceived activity limitation caused by back pain was measured using a precise Arabic translation of the Roland-Morris Back Pain Disability Questionnaire (RM). ${ }^{31}$ Derived from the Sickness Impact Profile, the RM contains 24 items relating to the influence of back pain on many activities of daily living. The individual scores may be converted to percentages ranging from $0 \%$ for "no disability" to $100 \%$ for "severe disability." The English language version of the RM has had excellent psychometric properties for detecting meaningful change in patient status. ${ }^{32}$

Measurement of Lumbar Extensor Force. Before and after intervention, all subjects completed an isometric lumbar extension strength (ILES) test using the $\mathrm{MedX}^{\mathrm{TM}}$ (Ocala, FL) lumbar extension machine. ${ }^{33,34}$ This procedure is described by Graves et $a l^{33}$ and has yielded reliable measures. ILES was determined by computing the mean value of peak torque tested at 7 lumbar spine angles: $0^{\circ}, 12^{\circ}, 24^{\circ}, 36^{\circ}, 48^{\circ}, 60^{\circ}$, and $72^{\circ}$.

\section{Intervention}

Lumbar Extensor Strengthening Protocol. Subjects were instructed in dynamic training techniques using the protocol described by Graves ${ }^{34,35}$ and Pollock ${ }^{36}$ et al. All training sessions were conducted by a physical therapist who had considerable experience using the MedX ${ }^{\mathrm{TM}}$ machine. Training consisted of variable resistance exercise using the $\mathrm{MedX}^{\mathrm{TM}}$ machine with a workload equal to $50 \%$ of the peak isometric torque obtained during preintervention isometric testing. The training was conducted once per week for 10 weeks. Initially, a 5-minute 
Table 1. Procedures Used for Physical Performance Testing*

\begin{tabular}{ll}
\hline Task & \multicolumn{1}{c}{ Procedure } \\
\hline $\begin{array}{c}\text { Modified sit to stand } \\
\text { (from the floor) }\end{array}$ & $\begin{array}{l}\text { This test started with the subject sitting } \\
\text { on the floor with his or her legs } \\
\text { crossed. The subject was instructed to } \\
\text { stand up until the complete upright } \\
\text { posture was achieved and then to sit } \\
\text { back again on the floor, returning to } \\
\text { the starting position as quickly and } \\
\text { safely as possible. The task was } \\
\text { repeated } 10 \text { times, ending in standing } \\
\text { position. } \\
\text { This test started with the subject } \\
\text { standing. The subject was instructed to } \\
\text { bend forward as far as he or she was } \\
\text { able, without bending the knees, and } \\
\text { then to extend the trunk to starting } \\
\text { erect posture as quickly and safely as } \\
\text { possible. The movement was repeated } \\
10 \text { times, ending in standing. } \\
\text { The subject was instructed to walk a } \\
\text { distance of } 30 \text { m as fast and safely as } \\
\text { possible. }\end{array}$ \\
Fast walking &
\end{tabular}

* A stopwatch was used to time each task.

warm-up session was performed, after which the subjects were instructed to perform one set of variable resistance lumbar extension exercises through the available lumbar spine range of motion $\left(0^{\circ}-72^{\circ}\right)$. A load was selected that allowed 6 to 12 repetitions before fatigue. Progressive resistance training was achieved by increasing the load by approximately $5 \%$ when at least 12 repetitions could be completed. Repetitions were performed in a slow and controlled manner. For each subject, the training load and repetitions completed during the dynamic training session were recorded. The subjects were instructed not to alter normal daily activities, life-style, or diet during the treatment. No instruction for home exercises or activity guidelines was provided.

Statistical Analysis. Data were coded and entered into a database. Analyses were performed using SPSS Base, Regression and Advanced software (version 10.0, SPSS Inc., Chicago, Ill). Descriptive summary statistics, independent $t$ tests, and $\chi^{2}$ analyses were used to summarize data and determine the presence of gender-based differences. The degree of association between the study measures was assessed using a bivariate correlation matrix. Outcome was determined by the magnitude of change in the RM score. Those subjects whose postintervention RM score reflected a decrease of greater than, or equal, to the minimal detectable change score of $16 \%$ were classified as "improved." 32 The remaining subjects were classified as "not improved." Intention-to-treat analyses were used to account for those subjects who dropped out by using their initial scores as their final scores, therefore, classifying them as "not improved." Independent $t$ tests were used to determine the differences in preintervention measures between those subjects classified as "improved" or "not improved" after intervention. Differences for each of the self-report and physical performance measures obtained before and after the intervention were determined using paired $t$ tests. Confidence intervals and effect sizes were calculated. The formula used to calculate effect size was: (mean pretest - mean of post-test)/standard deviation (SD) of mean of pretest. Bivariate correlations were performed for those variables reflecting significant differences to determine their association with the change in the RM score following treatment (RM preintervention - RM postintervention).

To determine the validity indexes provided by a range of scores on the subscales of the FABQP, a visual assessment of the data was performed using cross tabulation to identify natural cutoff points. A $2 \times 3$ contingency table was formed to compare ranges of test scores by outcome. This was then reduced to a $2 \times 2$ contingency table to reflect better the difference in scores. Sensitivity, specificity, and likelihood ratios and their $95 \%$ CI were calculated. ${ }^{37,38}$

\section{Results}

\section{Sample Characteristics}

There were 42 subjects initially enrolled in the study. A total of 36 completed the entire intervention and testing protocol. The initial sample was comprised of 22 males and 20 females. The mean age of the male group $(45.0$

Table 2. Bivariate Correlations Between Subject Characteristics, Self-Report, and Performance Measures Obtained Before Intervention*

\begin{tabular}{|c|c|c|c|c|c|c|c|c|c|c|c|c|}
\hline & Age & BMI & $\begin{array}{l}\text { Days of } \\
\text { Activity } \\
\text { Limitation }\end{array}$ & $\begin{array}{c}\text { Pain } \\
\text { Duration }\end{array}$ & FABQP & FABOW & $\begin{array}{l}\text { Overall } \\
\text { Pain }\end{array}$ & ILES & $\begin{array}{c}\text { AP } \\
\text { Sit-Stand }\end{array}$ & $\begin{array}{c}\text { AP } \\
\text { Forward } \\
\text { Bending }\end{array}$ & $\begin{array}{c}\text { AP } \\
\text { Fast Walk }\end{array}$ & RM \\
\hline Age & $X X$ & $0.54 \ddagger$ & -0.04 & 0.12 & -0.07 & $-0.51 \ddagger$ & 0.28 & 0.17 & -0.15 & $-0.40 \dagger$ & $-0.39 \dagger$ & $0.33 \dagger$ \\
\hline BMI & & $X X$ & -0.26 & 0.30 & -0.10 & -0.20 & $0.31 \dagger$ & 0.47 & -0.08 & -0.26 & -0.24 & $0.59 \ddagger$ \\
\hline $\begin{array}{l}\text { Days of activity } \\
\text { limitation }\end{array}$ & & & $\mathrm{XX}$ & -0.13 & -0.01 & .016 & -0.14 & -0.28 & 0.26 & 0.14 & -0.10 & $-0.38 \dagger$ \\
\hline Pain duration & & & & $X X$ & -0.07 & -0.02 & -0.02 & $0.32 \dagger$ & -0.04 & -0.06 & 0.06 & $0.35 \dagger$ \\
\hline FABQP & & & & & $X X$ & -0.48 & $0.31 \dagger$ & 0.30 & 0.25 & $0.34 \dagger$ & 0.32 & 0.18 \\
\hline FABOW & & & & & & XX & 0.03 & -0.13 & 0.06 & 0.11 & 0.14 & -0.20 \\
\hline Overall pain & & & & & & & $X X$ & 0.18 & 0.11 & -0.25 & -0.15 & $0.35 \dagger$ \\
\hline ILES & & & & & & & & $X X$ & 0.18 & -0.12 & -0.20 & $0.56 \ddagger$ \\
\hline AP sit to stand & & & & & & & & & $X X$ & $0.35 \dagger$ & $0.34 \dagger$ & -0.04 \\
\hline AP forward bending & & & & & & & & & & $X X$ & $0.90 \ddagger$ & -0.16 \\
\hline AP fast walk & & & & & & & & & & & $X X$ & -0.11 \\
\hline $\mathrm{RM}$ & & & & & & & & & & & & $X X$ \\
\hline
\end{tabular}

${ }^{*} \mathrm{~N}=42$.

$+P<0.05$

$\neq P<0.01$

$\mathrm{AP}=$ anticipated pain; $\mathrm{BMI}=$ body mass index 
Table 3. Differences in Preintervention Measures Between Those Subjects With Clinically Meaningful and Not Clinically Meaningful Improvement in the RM Score After Intervention

\begin{tabular}{|c|c|c|c|c|c|}
\hline Measure & Positive Outcome (mean) & Negative Outcome (mean) & Difference & $95 \% \mathrm{Cl}$ & $P$ Value \\
\hline No. of subjects & 17 & 25 & & & \\
\hline Age & $42.5(7.8)$ & $42.1(5.9)$ & -0.45 & -5.0 to -4.1 & NS \\
\hline $\mathrm{BMI}$ & $28.2(2.9)$ & $28.7(3.3)$ & 0.44 & $-1.5-2.4$ & NS \\
\hline Days of reduced activity & $18.1(12.1)$ & $14.1(8.7)$ & -3.9 & $-10.9-2.9$ & NS \\
\hline Duration of pain (mos) & $4.4(2.3)$ & $5.2(2.1)$ & 0.85 & $-0.58-2.3$ & NS \\
\hline FABOP & $21.0(6.7)$ & $26.8(4.4)$ & 5.8 & $2.0-9.6$ & 0.002 \\
\hline FABOW & $35.1(13.4)$ & $33.0(13.3)$ & -2.1 & $-10.7-6.5$ & NS \\
\hline Overall pain (mm) & $71.1(14.0)$ & $80.0(10.9)$ & 8.7 & $0.47-17.0$ & 0.039 \\
\hline ILES & 96.3 & 102.2 & 5.8 & $2.41-14.1$ & NS \\
\hline
\end{tabular}

NS $=$ not significant.

years, $S D=6.2$ ) was higher than that of the female group (39.25 years, $\mathrm{SD}=5.8 ; P=0.03)$. At the time of entry into the study, 27 subjects were employed on a full-time basis, 6 were employed part-time, 1 subject was a homemaker, and the remaining 8 were retired. Males were more likely to be employed full-time, whereas females were more likely to be retired $(P=0.029)$. Females reported a higher number of days of activity limitation caused by back pain than did males before beginning the study (20.8 vs. 11.1 days, $P=0.002)$. Preintervention pain duration and intensity were not significantly different between males and females. The average duration of pain was 4.8 months $( \pm 2.20)$, with a range of $2-9$ months. The overall pain intensity reported during initial testing was moderately high (mean $=76.4 \mathrm{~mm}$, range 48.0-98.0). Females had lower RM scores than males before intervention $(71 \%$ vs. $86 \%$; $95 \%$ CI of difference $=4 \%$ to $24 \%, P=0.006)$ and after intervention $(52 \%$ vs. $71 \% ; 95 \% \mathrm{CI}=8 \%$ to $30 \%, P=0.001)$. Nine of 20 females and 8 of 22 males were classified as "improved." There were no gender-based differences in FABQP, FABQW, or anticipated pain.

\section{Correlations of Preintervention Measures}

The FABQP had a significant but low correlation with overall pain $(\mathrm{r}=0.31)$ and with anticipated pain before the forward bending test $(r=0.34)$ (Table 2). The FABQW was negatively correlated with age $(\mathrm{r}=-0.51)$. Anticipated pain before forward bending and fast walking were also negatively correlated with age $(\mathrm{r}=-0.40$ and -0.39 , respectively). Anticipated pain before each of the 3 performance tests was correlated with each of the other 2 values of anticipated pain. Neither anticipated pain nor the subscales of the FABQ were significantly correlated with body mass index, days of activity limitation, pain duration, or lumbar muscle strength. There were moderate positive correlations between the preintervention RM score with body mass index $(\mathrm{r}=0.59)$ and ILES $(\mathrm{r}=0.56)$. There were moderate-to-weak positive correlations among the preintervention RM score and age $(\mathrm{r}=0.33)$, pain duration $(\mathrm{r}=0.35)$ and overall pain $(\mathrm{r}=0.35)$.

\section{Preintervention Measures Associated with Improvement in the Roland-Morris Score}

The group mean of the preintervention RM scores was moderately high $(78.7 \% \mathrm{SD}=16.9, \mathrm{n}=42)$. Using intention-to-treat strategies, statistically significant improvements were noted for the group mean after treatment with a postintervention mean of $62.3 \%, \mathrm{SD}=19.4$ $(95 \% \mathrm{CI}=11.0 \%$ to $21.5 \%, P=0.00)$. The effect size was 0.96 . Because the lower value in the confidence interval was below the minimal detectable change score for the RM of $16 \%$, it cannot be stated with certainty that the mean degree of improvement was clinically meaningful. When the postintervention group was dichotomized based on the presence or absences of a $16 \%$ reduction in the RM score, 17 subjects were classified as "improved" and 25 as "not improved." Table 3 illustrates the differences in preintervention scores between these 2 groups. The mean scores of the preintervention FABQP and the preintervention rating of overall pain were significantly higher for those subjects who did not improve compared to those who did. There were no other significant differences between the 2 groups.

Bivariate correlations between the preintervention measures of the FABQP and overall pain with the change score of the RM revealed moderate but significant negative correlations $(-0.51$ and -0.35 , respectively). This

Table 4. Validity Indexes for the High Scores $(\geq 29)$ on the FABOP Compared to Low Scores $(10-\leq 20)$

\begin{tabular}{|c|c|c|}
\hline Test & $\begin{array}{l}\text { No Meaningful Clinical } \\
\text { Improvement of RM } \\
\text { Score (Lack of } \\
\text { Clinically Meaningful } \\
\text { Outcome) }\end{array}$ & $\begin{array}{l}\text { Meaningful Clinical } \\
\text { Improvement of RM } \\
\text { Score (Clinically } \\
\text { Meaningful Outcome) }\end{array}$ \\
\hline $\begin{array}{l}\text { FABO } \geq 29 \text { (high score) } \\
F A B O \leq 10 \text { (low score) }\end{array}$ & $\begin{array}{r}13 \\
2\end{array}$ & $\begin{array}{l}2 \\
7\end{array}$ \\
\hline \multicolumn{3}{|c|}{$\begin{array}{l}\text { Sensitivity of high score to identify correctly those subjects with a lack of } \\
\text { clinically meaningful outcome }=0.87(95 \% \mathrm{Cl} 0.70-1.0) \text {. } \\
\text { Specificity of low score to identify correctly those subjects with a clinically } \\
\text { meaningful outcome }=0.77(95 \% \mathrm{Cl} 0.45-1.0) \\
\text { Likelihood ratio of a high score to increase the pretest probability of a lack of } \\
\text { clinically meaningful outcome }=3.78(95 \% \mathrm{Cl} 2.4-5.16) \\
\text { Likelihood ratio of a low score to increase the pretest probability of a clinically } \\
\text { meaningful outcome }=0.15(95 \% \mathrm{Cl} 0.48-0.78)\end{array}$} \\
\hline
\end{tabular}


Table 5. Differences in Preintervention Measures of Anticipated Pain, Reported Pain, and Time to Complete Physical Performance Testing for Those Subjects with Clinically Meaningful and Not Clinically Meaningful Improvement in the RM Score After Intervention

\begin{tabular}{|c|c|c|c|c|c|}
\hline Measure & Positive Outcome (mean) & Negative Outcome (mean) & Difference & $95 \% \mathrm{Cl}$ & $P$ Value \\
\hline No. of subjects & 17 & 25 & & & \\
\hline \multicolumn{6}{|l|}{ Sit to stand } \\
\hline $\mathrm{AP}(\mathrm{mm})$ & $70.8(8.1)$ & $69.6(12.3)$ & -1.3 & $-7.6-5.1$ & NS \\
\hline Reported pain (mm) & $57.0(11.1)$ & $55.6(9.8)$ & -1.4 & $-8.1-5.4$ & NS \\
\hline Time $(s)$ & $41.9(7.2)$ & $41.5(7.1)$ & -0.28 & $-5.2-4.6$ & NS \\
\hline \multicolumn{6}{|l|}{ Forward bending } \\
\hline $\mathrm{AP}(\mathrm{mm})$ & $82.6(9.9)$ & $85.5(9.9)$ & 2.9 & $-3.4-9.2$ & NS \\
\hline Reported pain (mm) & $69.0(13.1)$ & $65.3(12.9)$ & -3.7 & $-12.0-4.6$ & NS \\
\hline Time (s) & $29.5(4.0)$ & $32.3(4.6)$ & 2.8 & $-0.1-5.7$ & NS \\
\hline \multicolumn{6}{|l|}{ Fast walking } \\
\hline $\mathrm{AP}(\mathrm{mm})$ & $84.3(9.5)$ & $87.6(3.2)$ & 3.2 & $-2.8-9.3$ & NS \\
\hline Reported pain (mm) & $62.5(9.7)$ & $64.4(10.1)$ & 1.9 & $-4.4-8.2$ & NS \\
\hline Time (s) & $18.9(2.2)$ & $19.3(2.0)$ & 0.36 & $-1.1-1.8$ & NS \\
\hline
\end{tabular}

result implied that for our sample, higher preintervention scores for FABQP and/or overall pain were associated with lower change scores in the RM. To provide a richer understanding of this relationship, cross tabulation was used to compare visually the distribution of the scores on the FABQP between those subjects who improved versus those who did not. Natural cutoff points appeared at $\leq 20,21-28$, and $\geq 29$. Of those subjects with a preintervention FABQP of $\leq 20,7$ of 9 had improvement, while for those subjects with a preintervention score $\geq 29,13$ of 15 did not have improvement. For those subjects with a midrange score (21-28), there was no obvious trend ( 10 of 18 did not have improvement). A $2 \times 2$ contingency table was created, and validity indexes were calculated. A "high score" $(\geq 29)$ was considered a positive $(+)$ test finding and a "low score" $(\leq 20)$, a negative (-) test finding (Table 4). The sensitivity of a high score to identify correctly those subjects with a lack of clinically meaningful outcome was 0.87 (95\% CI $=$ $0.70-1.0)$. The specificity of low score to identify correctly those subjects with a clinically meaningful outcome was 0.77 (95\% CI $=0.45-1.0)$. The positive likelihood ratio of a high score to increase the pretest probability of a lack of clinically meaningful outcome was 3.78 (95\% CI 2.4-5.16), while the negative likelihood ratio of a low score to increase the pretest proba- bility of a clinically meaningful outcome was 0.15 (95\% CI $0.48-0.78)$.

Table 5 summarizes the differences in outcome relative to anticipated pain and reported pain associated with physical performance testing before intervention. No significant differences were noted. The wide confidence intervals characterize the large variation between subjects relative to anticipated pain and reported pain. There were no significant correlations between anticipated pain and the subject's report of overall pain before any of the preintervention or postintervention physical performance tests $(r=-0.28-0.09)$. For those subjects who completed intervention, all measures of anticipated pain and reported pain improved (Table 6). However, in all comparisons, anticipated pain remained significantly higher than reported pain $(P<0.01)$.

\section{Discussion}

This study examined 2 constructs that may influence the clinical course of people with LBP: fear avoidance beliefs and anticipated pain. In our sample, we found that the high preintervention scores from the physical activity subscale of the FABQ were predictive of a lack of patient improvement. Our preliminary data suggest that the FABQP may be a useful screening tool to detect certain patients at increased risk for poor outcome when it is

Table 6. Preintervention and Postintervention Measures of Anticipated Pain and Reported Pain Rated 0- $100 \mathrm{~mm}$

\begin{tabular}{|c|c|c|c|c|}
\hline \multirow[t]{2}{*}{ Variable (U) } & \multirow{2}{*}{$\frac{\text { Mean Score }}{\text { Preintervention }}$} & \multirow{2}{*}{$\frac{\text { Mean Score }}{\text { Postintervention }}$} & \multirow[t]{2}{*}{$95 \% \mathrm{Cl}$ of Difference } & \multirow[t]{2}{*}{ Effect Size } \\
\hline & & & & \\
\hline AP: Sit to stand & 68.0 & 39.1 & $26.7-31.1$ & 2.92 \\
\hline RP: Sit to stand & 56.9 & 18.8 & $36.5-40.1$ & 3.97 \\
\hline AP: Forward bending & 84.0 & 50.7 & $31.2-36.3$ & 3.23 \\
\hline RP: Forward bending & 67.8 & 29.2 & $34.5-42.7$ & 2.86 \\
\hline AP: Fast walking & 85.7 & 51.9 & $32.3-35.3$ & 3.40 \\
\hline RP: Fast walking & 63.6 & 29.3 & $31.2-37.3$ & 3.34 \\
\hline
\end{tabular}


dichotomized into "high" and "low" scores, leaving a mid region of "inconclusive" scores. This result is consistent with its scale structure that includes 3 response zones: "disagree" (low), "unsure" (inconclusive), and "agree" (high score), implying that those with low scores do not believe that physical activity will harm them, those with high scores do, and those in the middle are not sure. Using the interpretation of likelihood ratios submitted by Jaeschke et al, ${ }^{39}$ the positive likelihood ratio of 3.78 for those subjects with $\geq 29$ on the FABQP represents a small but potentially important reduction in the preintervention likelihood of improvement. Because the negative likelihood ratio confidence interval includes zero, it is inconclusive, thus a low score on this instrument does not necessarily increase the preintervention likelihood of improvement.

In our sample, we found that scores from the FABQW were not predictive of patient outcome. This finding is in conflict with other reports. For example, Fritz et $a l,{ }^{24}$ when studying injured workers with acute LBP, reported that the FABQW was a greater predictor of delayed recovery than was the FABQP. Vowles and Gross ${ }^{27}$ reported that changes in the work specific fears following intervention were more important than pain and fear of physical activity for predicting physical capability to work. Because our study did not include individuals receiving worker's compensation, we believe that this illustrates population specific validity of these subscales. For example, previous studies sampling injured workers in the United States, a country with substantial workers' compensation and litigation related to LBP, reported that high scores on the work subscale of the FABQ were more predictive of poor outcome than those from the physical activity subscale. ${ }^{24,25}$ In our sample of people who where not injured workers and who lived in a country that lacks workers' compensation and litigation for LBP, the work subscale of the FABQ was not predictive of outcome. However, very high scores on the physical activity subscale of the FABQ were predictive of poor outcome.

Interestingly, anticipated pain showed wide betweensubject variation and had a very limited association with either subscale of the FABQ. The preintervention measures of anticipated pain before forward bending had a significant but weak correlation with the preintervention FABQP $(\mathrm{r}=0.34)$. There were no other significant correlations of preintervention or postintervention measures of FABQ with anticipated pain, questioning the association of anticipated pain to fear avoidance beliefs in this population of people. This absence of a strong correlation between the subscales of the FABQ and anticipated pain is consistent with our previous work ${ }^{2}$ and suggests that these measures represent different aspects of the multidimensional pain experience. ${ }^{1-5,40-43}$

Our belief was that following intervention, the measures of anticipated pain and actual reported pain would approach one another as patients had overall improvement in strength as well as reductions in pain related to activity. Despite the significant degree of reduction in these measures following intervention, anticipated pain remained significantly higher than the actual reported pain. The reason for this is unclear and may reflect a learned "pain memory" by the subject.

Overall, anticipated pain did not seem to be strongly linked to any other subject factor that was measured in our study. There were no clear gender-based differences. The most consistent high correlations occurred between anticipated pain and actual reported pain for fast walking (preintervention comparisons $r=0.94$, postintervention comparisons $r=0.56$ ). Age had a moderate, negative correlation with anticipated pain before forward bending (preintervention comparisons $\mathrm{r}=-0.44$, postintervention comparisons $r=-0.38$ ) and fast walking (preintervention comparisons $r=-0.44$, postintervention comparisons $r=-0.39$ ), suggesting that younger subjects had higher degrees of anticipated pain than did older subjects. However, anticipated pain was not strongly correlated with the RM score. These findings suggest that in the population addressed in this study, anticipated pain does not necessarily predict a subject's ability or beliefs related to performing tasks (i.e., although one may have a high degree of anticipated pain before an activity, this does not specifically determine his or her perceived ability to perform this activity). Considering this result, the role of anticipated pain as a distinct variable whose ability to predict overall pain and function is uncertain.

The data presented must be considered within a scope of limitations. Although the standardized measures in this study (RM, the subscales of the FABQ) were translated directly from English to Arabic by individuals familiar with both cultures, it is uncertain to what degree cultural factors influenced subject responses. ${ }^{44,45}$ None of the subjects in this study were taking any prescription based pain medication. The choice of $a \geq 16 \%$ improvement represents a meaningful detectable change score and was used as a "gold standard" to determine validity indexes of the FABQP. The overall decision of clinically meaningful or lack of clinically meaningful outcome should, however, be linked to patient specific goals. Our findings present preliminary information regarding the measurement properties and scope of the subscales of the FABQ on population of individuals from a Middle Eastern culture. Further study is needed on other subpopulations.

\section{- Conclusions}

For a sample of people from a Middle Eastern culture undergoing exercise intervention for LBP for which they are not receiving workers' compensation, the preintervention $F A B Q P$ is predictive of a lack of a clinically meaningful outcome when the observed scores are $\geq 29$. We believe that very high scores on the pain subscale of the FABQ may indicate a "red flag" for clinicians and researchers, indicating that a given patient (or subject) may be at increased risk for poor outcome and may ben- 
efit from further assessment of pain-related beliefs and behavior. ${ }^{40-43}$ Despite significant improvements in all variables following intervention, anticipated pain remained significantly higher than reported pain during physical performance testing. However, this relationship did not influence patient outcome.

\section{- Key Points}

- High scores obtained from the preintervention FABQ physical activity subscale predicted a likelihood of failure to have meaningful improvement on the Roland-Morris Questionnaire.

- In this sample of patients from a Middle Eastern culture who were not receiving workers' compensation, the preintervention work subscale of the FABQ was not predictive of meaningful improvement on the Roland-Morris Questionnaire.

- Despite significant improvements following intervention, anticipated pain remained higher than reported pain during physical performance testing. - The lack of correlation of anticipated pain to overall pain, fear avoidance beliefs, and the Roland-Morris Questionnaire infers that anticipated pain represents a construct that is distinct, and should be measured separately and in reference to a specific task.

\section{References}

1. Al-Obaidi SM, Nelson R, Al-Awadhi S, et al. The role of anticipation and fear of pain in the persistence of avoidance behavior in patients with chronic low back pain. Spine 2000;25:1126-31.

2. Al-Obaidi SM, Al-Zoabi B, Al-Shuwaie N, et al. The influence of pain and pain-related fear and disability beliefs on walking velocity in chronic low back pain. Int J Rehabil Res 2003;26:101-8.

3. Feuerstein M, Beattie P. Biobehavioral factors affecting pain and disability in low back pain: Mechanisms and assessment. Phys Ther 1995;75:267-80.

4. Schultz I, Crook JM, Berkowitz J, et al. Biopsychosocial multivariate predictive model of occupational low back disability. Spine 2002;27:2720-5.

5. Pincus T, Burton AK, Vogel, et al. A systematic review of psychological factors as predictors of chronicity/disability in prospective cohorts of low back pain. Spine 2002;27:E109-20.

6. Hides JA, Stokes MJ, Saide GA, et al. Evidence of lumbar multifidus muscle wasting ipsilateral to symptoms in patients with acute/ subacute low back pain. Spine 1994;19:165-72.

7. Kader DW, Wardlaw D, Smith W. Correlation between the MRI changes in lumbar multifidus muscles and leg pain. Clin Radiol 2000;55:145-9.

8. Addison R, Schultz A. Trunk strength in patients seeking hospitalization for chronic low back pain. Spine 1980;5:539-44.

9. Waddell G, Sommerville D, Henderson I. Objective clinical evaluation of physical impairment in chronic low back pain. Spine 1992;17:617-28.

10. Beattie P, Rothstein JM, Lamb RL. Reliability of the attraction method for measuring lumbar spine backward bending. Phys Ther 1987;67:364-6.

11. Crombez G, Vervaet L, Lysens R, et al. Avoidance and confrontation of painful, back-straining movements in chronic back pain patients. Behav Modif 1998;22:62-77.

12. Crombez G, Vlaeyen JW, Heuts PH, et al. Pain-related fear is more disabling than pain itself: Evidence on the role of pain-related fear in chronic back pain disability. Pain 1999;80:329-39.

13. Dolce JJ, Crocker MF, Doleys DM. Prediction of outcome among chronic pain patients. Behav Res Ther 1986B;24:313-9.

14. Feuerstein M, Sult S, Houle M. Environmental stressors and chronic low back pain: Life events, family and work environment. Pain 1985;22:295307.

15. Hazard RG, Fenwick JW, Kalisch SM, et al. Functional restoration with behavioral support: A one year prospective study of patients with chronic low back pain. Spine 1989;14:157-61.

16. Johnson JE. Effects of accurate expectations about sensations on the sensory and distress components of pain. J Pers Soc Psychol 1973;27:261-75.

17. Johnson JE, Rice VH. Sensory and distress components of pain: Implications for the study of clinical pain. Nurs Res 1974;23:203-9.

18. Lethem J, Slade PD, Troup JD, et al. Outline of a fear-avoidance model of exaggerated pain perception-I. Behav Res Ther 1983;21:401-8.

19. Riley JF, Ahern DK, Follick MJ. Chronic pain and functional impairment: Assessing beliefs about their relationship. Arch Phys Med Rehabil 1988;69: $579-82$.

20. Slade PE, Troup JD, Lethem J, et al. The fear avoidance model of exaggerated pain perception-II. Preliminary studies of coping strategies for pain. Behav Res Ther 1983;21:409-16.

21. Vlaeyen JW, Crombez G. The role of fear of movement (re)injury in pain disability. J Occup Rehab 1995;5:235-52.

22. Vlaeyen JW, Crombez G. Fear of movement/(re)injury, avoidance and pain disability in chronic low back pain patients. Man Ther 1999;4:187-95.

23. Waddell G, Newton M, Henderson I, et al. A Fear-Avoidance Beliefs Questionnaire (FABQ) and the role of fear-Avoidance beliefs in chronic low back pain and disability. Pain 1993;52:157-68.

24. Fritz JM, George SZ, Delitto A. The role of fear-avoidance beliefs in acute low back pain: Relationships with current and future disability and work status. Pain 2001;94:7-15.

25. Fritz J, George SZ. Identifying psychosocial variables in patients with acute work-related low back pain: The importance of fear avoidance beliefs. Phys Ther 2002;82:973-83.

26. Verbunt JA, Seelen HA, Vlaeyen JW, et al. Fear of injury and physical deconditioning in patients chronic low back pain. Arch Phys Med Rehabil 2003;84:1227-32.

27. Vowles KE, Gross RT. Work-related beliefs about injury and physical capability for work in individuals with chronic pain. Pain 2003;101:291-8.

28. Price DD, McGrath PA, Rafii A, et al. The validation of visual analogue scales as ratio scale measures for chronic and experimental pain. Pain 1983; 17:45-56.

29. Revill SI, Robinson JO, Rosen M, et al. The reliability of a linear analogue for evaluating pain. Anaesthesia 1976;31:1191-8.

30. Simmonds M, Olson S, Jones S, et al. Psychometric characteristics and clinical usefulness of physical performance tests in patients with low back pain. Spine 1998;23:2412-21.

31. Roland M, Morris, R. A study of the natural history of back pain. Part I: Development of a reliable and sensitive measure of disability in low-back pain. Spine 1983;8:141-4.

32. Stratford PW, Binkley J, Solomon P, et al. Defining the minimum level of detectable change for the Roland-Morris questionnaire. Phys Ther 1996;76: $359-65$.

33. Graves JE, Pollock ML, Carpenter DM, et al. Quantitative assessment of full range of motion isometric lumbar extension strength. Spine 1990;15:289-94.

34. Graves JE, Pollock ML, Foster D, et al. Effect of training frequency and specificity on isometric lumbar extension strength. Spine 1990;15:504-9.

35. Graves JE, Webb DC, Pollock ML, et al. Pelvic stabilization during resistance training: Its effect on the development of lumbar extension strength. Arch Phys Med Rehabil 1994;75:210-5.

36. Pollock ML, Leggett SH, Graves JE, et al. Effect of resistance training on lumbar extension strength. Am J Sports Med 1989;17:624-9.

37. Riddle DL, Stratford PW. Interpreting validity indexes for diagnostic tests: An illustration using the Berg Balance Test. Phys Ther 1999;79:939-48.

38. Simel DL, Samsa GP, Matchar DB. Likelihood ratios with confidence intervals: Sample size estimation for diagnostic test studies. J Clin Epidemiol 1991;44:763-70.

39. Jaeschke R, Guyatt GH, Sackett DL. Users' guides to the medical literature. III. How to use an article about a diagnostic test. B. What are the results and will they help me in caring for my patients? The Evidence-Based Medicine Working Group. JAMA 1994;271:703-7.

40. Holroyd KA, Talbot F, Holm JE, et al. Assessing the dimensions of pain: A multitrait-multimethod evaluation of seven measures. Pain 1996;67:259-65.

41. Linton SJ. Early identification and intervention in the prevention of musculoskeletal pain. Am J Ind Med 2002;41:433-42.

42. Proctor T, Gatchel RJ, Robinson RC. Psychosocial factors and risk of pain and disability. Occup Med 2000;15:803-12.

43. Turk DC. The role of psychological factors in chronic pain. Acta Anaesthesiol Scand 1999;43:885-88.

44. Guillemin F, Bombardier C, Beaton D. Cross-cultural adaptation of healthcare related quality of life measures: Literature review and proposed guidelines. J Clin Epidemiol 1993;46:1417-32.

45. Guillemin F. Measuring health status across cultures. Eur J Rheumatol Inflamm 1995;2(suppl):102-3. 